\title{
Quantitative Analysis of the Brownian Motion of Small Liquid Lead Inclusions in Solid Aluminum
}

\author{
U. Dahmen, T. Radetic, J. Turner, S. Prokofjev*, M.T. Levinsen** and E. Johnson** \\ National Center for Electron Microscopy, LBNL, Berkeley, 94720 CA, USA \\ *Dept. of Solid State Physics, Russian Academy of Sciences, Chrenogolovka, Russia \\ **Niels Bohr Institute, University of Copenhagen, Denmark
}

The thermal motion of liquid inclusions or gas bubbles in solids is of fundamental and technological interest. For example, liquid migration in a thermal gradient has been used for semiconductor doping, and the rate of thermal drift indicates the mechanism of transport [1]. Liquid Pb inclusions in $\mathrm{Al}$ a few nanometers, in size have been observed to undergo random motion during in-situ heating in a transmission electron microscope [2]. The velocity and type of motion are a strong function of size and temperature. In this work, we have made direct real-time electron microscopy observations of the random motion of nanoscale liquid $\mathrm{Pb}$ inclusions in solid $\mathrm{Al}$ at temperatures around $450^{\circ} \mathrm{C}$. Particle migration was recorded on video tape and analyzed frame by frame to determine the precise position of particles relative to a fixed reference frame. The resulting data sets were used to analyze the statistical properties of the particle motion and to obtain quantitative measures of the distribution of step sizes, the fractal dimension of the path of migration and the particle diffusion coefficient.

Figure 1 shows a still micrograph of an $\mathrm{Al}$ foil containing a field of liquid $\mathrm{Pb}$ inclusions at a temperature of $423^{\circ} \mathrm{C}$ (the bulk melting point of $\mathrm{Pb}$ is $327^{\circ} \mathrm{C}$ ). The larger inclusions display $\{111\}$ facets due to the anisotropy of the interfacial energy between liquid $\mathrm{Pb}$ and solid $\mathrm{Al}$ [3]. At a slightly higher temperature, smaller liquid inclusions such as the one marked by an arrow begin to migrate in random directions. We followed the migration of such particles for extended periods of time and recorded their motion on video tape. A few superimposed images from a sequence recorded at $438^{\circ} \mathrm{C}$ are shown in figure 2. Quantitative data could be extracted by digitizing image sequences from video recordings and processing them frame by frame using stereological image analysis. After choosing suitable sequences of about 1000 video frames, regions containing inclusions of interest were extracted and subjected to a series of image processing steps including background fitting, Gaussian convolution and thresholding. Subsequently, the position of each inclusion was determined by measuring the center of gravity, and the results exported to a spreadsheet for further analysis.

The superimposed images shown in figure 2 were taken from a sequence of 1056 frames and indicate that the motion was not unidirectional. To illustrate the random nature of the migration, the $\mathrm{x}$ and $\mathrm{y}$-components of the path are plotted separately in figure 3. For Brownian motion, each of these traces will independently exhibit random walk characteristics. A test for randomness of the $\mathrm{x}$ and y components is the statistical distribution of their displacements, shown in histograms in Figure 4. As expected for a random walk, both distributions are centered on a zero mean and closely follow Gaussian statistics. Similar behavior is found for larger time steps, albeit with a larger standard deviation. This increase in standard deviation is a measure of the mean square displacement. By plotting the mean square displacement as a function of time, we can obtain the diffusion coefficient for a single inclusion at a given temperature. Making such measurements on individual particles of known size for a series of temperatures will allow us to obtain the activation energy of the particle diffusion process [3].

\section{References}

[1] H.E. Cline and T.R. Anthony, J. Appl. Phys. 47, 2325 (1976) 
[2] B. Schmidt, Ph.D. thesis, University of Copenhagen (1992)

[3] H. Gabrisch, L. Kjeldgaard, E. Johnson and U. Dahmen, Acta. Mat. 49, 4259 (2001)

[4] This work was supported by the Director, Office of Science, Office of Basic Energy Sciences, Materials Science Division, US Department of Energy, under contract DE-AC3-76SF00098.

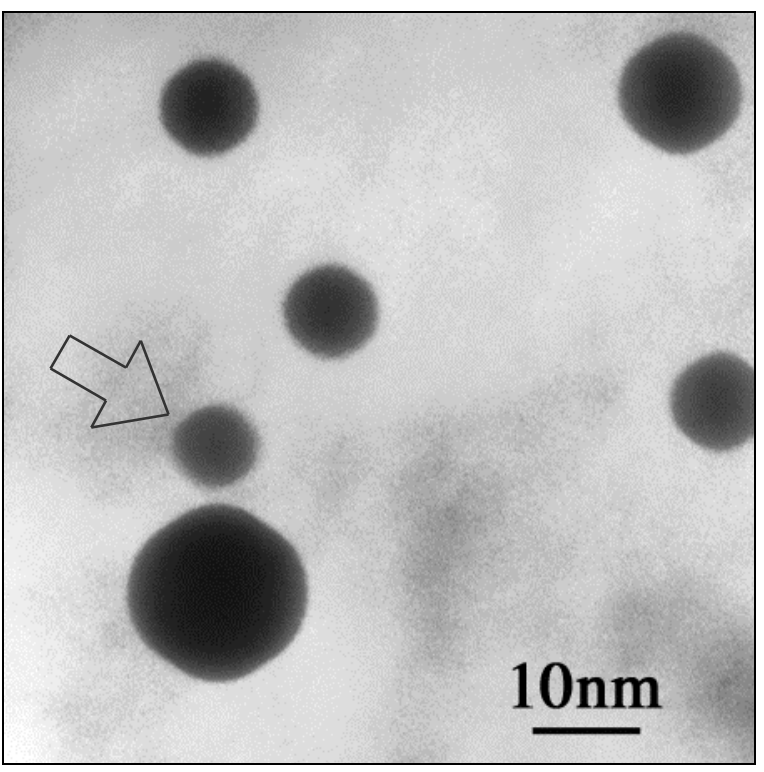

FIG. 1. Bright field micrograph of liquid $\mathrm{Pb}$ inclusions in solid $\mathrm{Al}$ at $423^{\circ} \mathrm{C}$. Inclusions such as the one marked with an arrow begin to migrate above $420^{\circ} \mathrm{C}$.

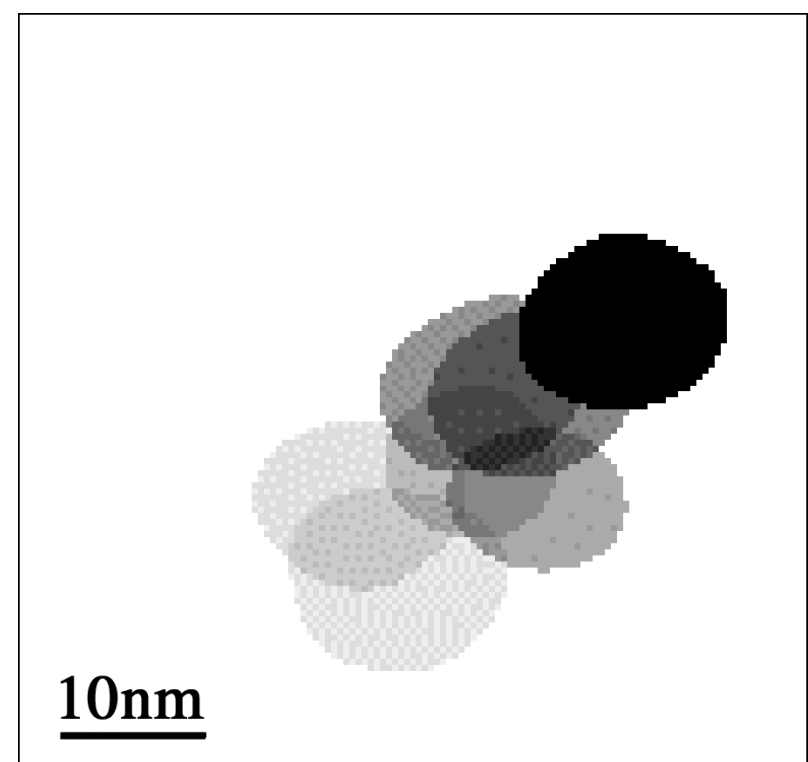

FIG. 2. Superposition of several frames extracted from a video sequence recorded during in-situ observation of Brownian motion of an individual inclusion at $438^{\circ} \mathrm{C}$.

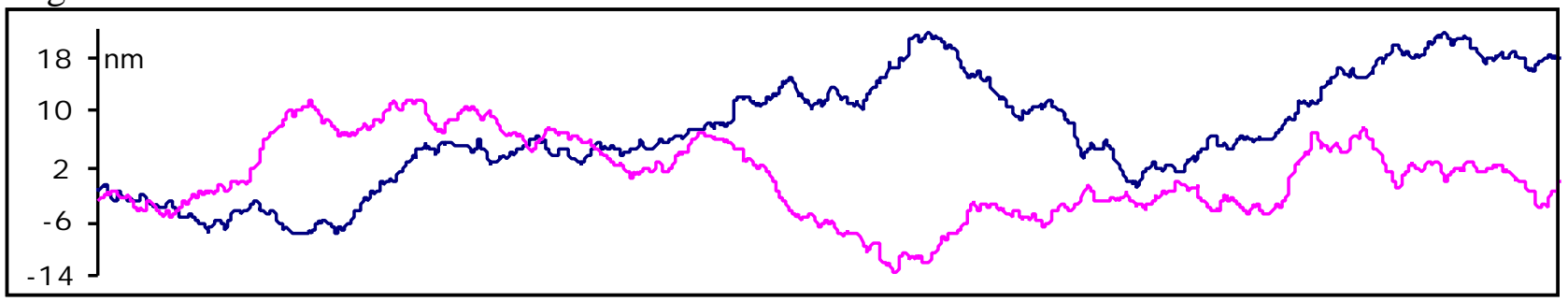

FIG. 3. Trace of $\mathrm{x}$ and $\mathrm{y}$ components of particle motion followed over 1056 video frames showing behavior typical of random walk.
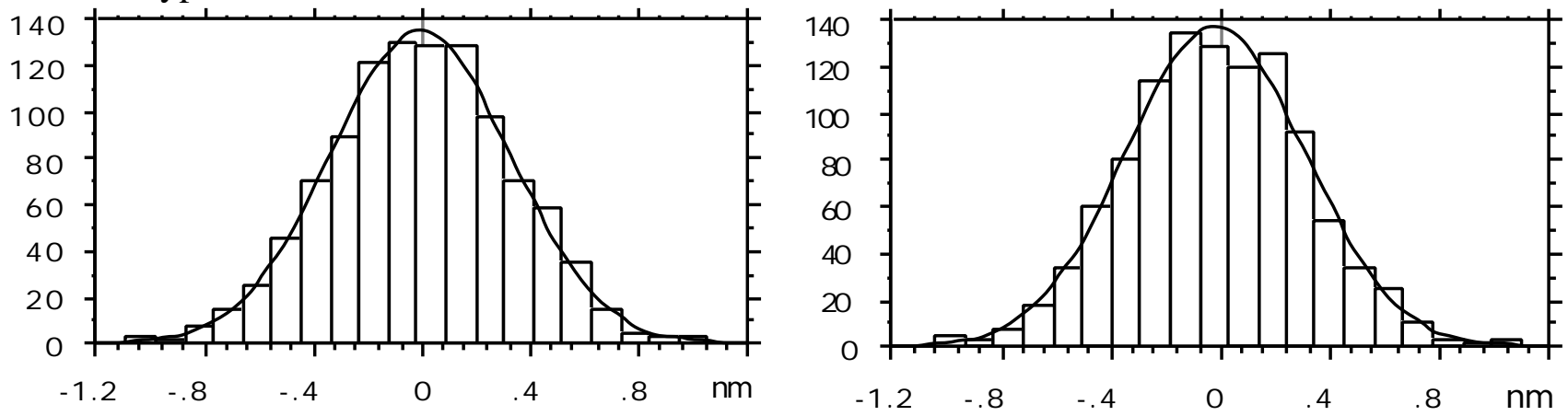

FIG. 4. Histograms showing statistical distribution of displacements measured separately in $\mathrm{x}$ and $\mathrm{y}$ directions indicating that the mean displacement is zero and the distributions are Gaussian (see least squares fit). 